Arq. Bras. Med. Vet. Zootec., v.56, n.3, p.346-354, 2004

\title{
Dinâmica folicular e momento da ovulação em vacas não lactantes das raças Gir e Nelore durante duas estações do ano
}

[Follicular dynamic and ovulation time of non-lactating Gir and Nelore cows during two seasons of the year]

\author{
A.M. Borges ${ }^{1}$, C.A.A. Torres $^{2}$, V.R. Rocha Júnior ${ }^{3}$, J.R.M. Ruas ${ }^{4}$, \\ M.M. Gioso ${ }^{5}$, J.F. Fonseca ${ }^{6}$, G.R. Carvalho ${ }^{2}$, V.V. Maffili ${ }^{6}$ \\ ${ }^{1}$ Escola de Veterinária da UFMG \\ Caixa Postal 567 \\ 30123-970 - Belo Horizonte, MG \\ ${ }^{2}$ Universidade Federal de Viçosa \\ ${ }^{3}$ Universidade Estadual de Montes Claros - Salinas, MG \\ ${ }^{4}$ Empresa de Pesquisa Agropecuária de Minas Gerais - Viçosa, MG \\ ${ }^{5}$ Aluno de Doutorado - Faculdade de Medicina Veterinária - UNESP - Botucatu, SP \\ ${ }^{6}$ Embrapa - Gado de Leite - Juiz de Fora, MG \\ ${ }^{7}$ Aluno de Doutorado - Universidade Federal de Viçosa, MG
}

\section{RESUMO}

Estudou-se o efeito da estação do ano na dinâmica folicular e no momento de ovulação utilizando-se 25 vacas não lactantes da raça Gir (10 no inverno e 15 no verão) e 15 vacas não lactantes da raça Nelore (sete no inverno e oito no verão), todas com ciclo estral normal. Após a detecção do estro, as vacas foram submetidas a exames ultra-sonográficos a cada seis horas com a finalidade de monitorar o momento de ovulação e, posteriormente, uma vez ao dia durante todo o ciclo estral, sendo monitorado um ciclo estral por animal e por estação do ano. As características foliculares não foram afetadas pela estação do ano e não diferiram entre as raças. Houve maior percentagem de ciclos com três $(68,0$ e $66,7 \%)$ e duas ondas foliculares $(24,0$ e $26,7 \%)$ nas raças Gir e Nelore, respectivamente. Os intervalos entre estros e entre ovulações foram menores para ciclos com duas ondas foliculares. A manifestação do estro ocorreu mais nas primeiras horas da manhã $(57,3 \%)$ e no final da tarde e início da noite $(28,0 \%)$. O momento de ovulação ocorreu 23,1 $\pm 5,6$ horas após a detecção do estro nas duas raças.

Palavras-chave: vaca, zebu, estação do ano, folículos, ovulação

\begin{abstract}
Season effect on follicular dynamic and ovulation time were studied on 25 non-lactating Gir cows (10 cows in the winter and 15 in the summer) and 15 non-lactating Nelore cows (7cows in the winter and 8 in the summer) exhibiting normal estrus cycles. After estrus detection cows were submitted to ultrasound examination every six hours to determine the time of ovulation, and after that, the examinations were performed once-a-day throughout the estrus cycle for each season. No effect of breed and season on follicular characteristics was observed. The follicular dynamic was characterized by the predominance of three (68.0 and 66.7\%) and two (24.0 and 26.7\%) follicular waves for Gir and Nelore cows, respectively. The estrus-to-ovulation intervals were shorter for cycles with two follicular waves. Most estrus signs were detected in the morning (57.3\%) and late afternoon (28.0\%). On average, Gir and Nelore cows ovulated 23 hours after estrus onset.
\end{abstract}

Keywords: cow, zebu, follicle, ovulation, season

Projeto parcialmente financiado pela FAPEMIG

Recebido para publicação em 2 de maio de 2003

Recebido para publicação, após modificações, em 4 de fevereiro de 2004

E-mail: alanmborges@vet.ufmg.br 


\section{INTRODUÇÃO}

O desenvolvimento de ondas foliculares acontece nos vários estádios reprodutivos dos bovinos. Exames ultra-sonográficos diários permitem verificar o início das ondas quando os folículos apresentam diâmetros iniciais entre 4 e $6 \mathrm{~mm}$. O processo de contínuo crescimento e regressão folicular é conhecido por dinâmica folicular e foi bem estudado por Ginther et al. (1989), Driancourt (1991), Fortune (1993) e Ginther (2000).

A dinâmica folicular em animais zebuínos, ainda pouco estudada, tem-se mostrado diferente da de bovinos de raças européias, de modo que o diâmetro dos folículos dominantes (Figueiredo et al., 1995) e a área do corpo lúteo (Pathiraja et al., 1986) são menores nas fêmeas zebuínas.

As fêmeas zebus apresentam característica sazonal, com menor eficiência reprodutiva nos períodos de dias mais curtos (Randel, 1994). O anestro e os estros anovulatórios foram verificados em vacas da raça Brahman durante o inverno (Stahringer et al., 1990). Nessa estação, a função lútea e a onda pré-ovulatória de $\mathrm{LH}$ foram diferentes das observadas nos meses de verão (Rhodes et al., 1982). Existem poucas informações sobre as diferenças estacionais na dinâmica folicular de zebuínos (comprimento dos ciclos estrais, número de ondas foliculares/ciclo e distribuição de vacas com duas, três ou quatro ondas/ciclo estral) e há evidências de que a estação do ano não exerce influência sobre as características do desenvolvimento folicular (Zeitoun et al., 1996). Assim, o anestro e a menor fertilidade durante os meses de outono e inverno decorrem muito mais de influências do meio (nutrição) do que por alteração nas ondas foliculares (Randel, 1994), de modo que pode haver interpretação errônea do efeito da estação do ano na função reprodutiva.

O índice de fertilidade depende, em parte, do momento ideal de inseminação que, na prática, é difícil de ser determinado. Assim, o período da detecção do estro é utilizado como referência para a inseminação e, caso seja generalizado para as diversas raças, pode haver comprometimento dos índices de fertilidade do rebanho. Isto ocorre porque o momento de ovulação, a intensidade e a duração do estro em bovinos de raças zebuínas são tidos como menores do que os observados nas raças européias (Plasse et al., 1970). Além disso, a grande percentagem de animais que apresenta estro durante a noite e nas primeiras horas da manhã dificulta sua deteç̧ão pelos sistemas convencionais de manejo (Valle et al., 1994).

O objetivo deste estudo foi caracterizar a dinâmica folicular ovariana e o momento de ovulação em vacas das raças Gir e Nelore, em duas estações do ano (verão e inverno).

\section{MATERIAL E MÉTODOS}

O estudo compreendeu dois períodos do ano: junho a agosto de 2000 (inverno) e janeiro e fevereiro de 2001 (verão). Foram utilizadas 25 vacas da raça Gir (10 no inverno e 15 no verão) e 15 da raça Nelore (sete no inverno e oito no verão), multíparas, não lactantes, com escore de condição corporal entre 3 e 5 (Ferreira, 1990) e peso vivo médio para a raça Gir de $414 \mathrm{~kg}$ no inverno e $416 \mathrm{~kg}$ no verão; e de $459 \mathrm{~kg}$ no inverno e $494 \mathrm{~kg}$ no verão para a raça Nelore. Todos os animais foram previamente submetidos a exame ginecológico, utilizando-se aqueles sem alteração clínica ou reprodutiva e que estavam ciclando regularmente segundo observação prévia de dois ciclos estrais.

As vacas mantidas em pastagens formadas por capim braquiária receberam mistura mineral e água à vontade.

A manifestação de estro foi monitorada visualmente durante uma hora, três vezes ao dia: pela manhã ( 6 às $7 \mathrm{~h})$, ao meio-dia (12 às $13 \mathrm{~h})$ e à tarde (18 às 19h), com o auxílio de rufião. Foram acompanhados 20 e 30 estros nas vacas das raças Gir e 14 e 16 em Nelore no inverno e no verão, respectivamente.

Os exames ultra-sonográficos foram realizados por via transretal utilizando-se um aparelho portátil $^{1}$ acoplado a um transdutor linear de 5MHz. A avaliação da dinâmica folicular foi feita por um único operador que registrou os diâmetros máximos do maior e do segundo maior folículo presente em cada ovário. A partir da detecção do estro, os animais foram submetidos à ultra-sonografia em intervalos de seis horas, definindo-se o momento de ovulação como o intervalo médio entre a última avaliação em que o folículo esteve presente até a sua não detecção com posterior formação de corpo lúteo (Rhodes et al., 1995b). 
A emergência da onda folicular foi estabelecida como o primeiro dia em que se encontrou um folículo com diâmetro entre 4 e $5 \mathrm{~mm}$. Cada onda foi dividida em: fase de crescimento - desde o dia de detecção do folículo até o dia em que cessou o seu crescimento; fase estática - desde o último dia de crescimento até o início da fase de redução de seu diâmetro; e fase de regressão - entre o último dia do diâmetro estático até atingir o diâmetro de 4 ou $5 \mathrm{~mm}$ (Ginther et al., 1989). O folículo dominante (FD) de cada onda foi o de maior diâmetro. Considerou-se apenas um folículo subordinado, por onda, classificado como um dos que apareceram simultaneamente ao folículo dominante, de diâmetro imediatamente inferior ao FD e superior aos demais folículos e de menor persistência. $\mathrm{O}$ diâmetro do folículo dominante e do subordinado foi medido pela maior distância ( $\mathrm{mm})$ entre dois pontos da cavidade antral dos folículos a partir de $4 \mathrm{~mm}$. Quando o folículo atingiu seu diâmetro máximo, obteve-se a duração (dias) e calculou-se a taxa de crescimento $(\mathrm{mm} / \mathrm{dia})$. Obtiveram-se a duração (dias) e a taxa de atresia ou de regressão, esta última definida como a diferença dos diâmetros atribuída à redução diária nas medidas foliculares $(\mathrm{mm})$ até um valor entre $4 \mathrm{e}$ $5 \mathrm{~mm}$, dividido pelo número de dias. $\mathrm{O}$ dia da divergência folicular foi definido como aquele em que os folículos dominante e subordinado diferiram (continuar crescendo ou regredir) em suas curvas de crescimento. A partir daí, calculou-se a duração da dominância folicular, que correspondeu ao intervalo do dia da divergência até o dia do surgimento de uma nova onda folicular.

A temperatura ambiente foi monitorada diariamente nas duas estações do ano (inverno e verão), por meio de termômetro de máximas e mínimas e aparelho termohigrógrafo (temperatura e umidade) colocados no curral experimental. Os dados de pluviosidade foram coletados na estação meteorológica localizada na própria fazenda.

$\mathrm{Na}$ análise estatística utilizou-se o programa SAEG 8.0 (Sistema..., 1997), aplicando-se análise de variância para as variáveis foliculares. Usou-se o teste Student Newman Keuls para comparação de médias. Para os dados de temperatura e umidade relativa do ar foi feita a análise de variância.

\section{RESULTADOS E DISCUSSÃO}

As duas estações estudadas foram caracterizadas pelos parâmetros climáticos mostrados na Tab. 1, onde se verificaram diferenças na temperatura máxima, mínima e umidade relativa do ar entre as duas estações estudadas.

Tabela 1. Caracterização climática registrada no local experimental durante duas estações do ano

\begin{tabular}{lcc}
\hline & Estação 1 (inverno/2000) & Estação 2 (verão/2001) \\
\hline Temperatura máxima (inferior - superior) $\left({ }^{\circ} \mathrm{C}\right)$ & $19,5-32$ & $26-40$ \\
Média da temperatura máxima $\left({ }^{\circ} \mathrm{C}\right)$ & $27,6 \pm 2,1 \mathrm{a}$ & $34,3 \pm 3,5 \mathrm{~b}$ \\
Temperatura mínima (inferior - superior) $\left({ }^{\circ} \mathrm{C}\right)$ & $4-17$ & $19,5-22,5$ \\
Média da temperatura mínima $\left({ }^{\circ} \mathrm{C}\right)$ & $12,2 \pm 2,9 \mathrm{a}$ & $21,3 \pm 0,7 \mathrm{~b}$ \\
Umidade relativa máxima (inferior - superior) $(\%)$ & $75,0-95,0$ & $76,0-100,0$ \\
Umidade relativa mínima (inferior - superior) $(\%)$ & $28,0-64,0$ & $32,0-65,0$ \\
Média da umidade relativa (\%) & $61,0 \pm 6,0 \mathrm{a}$ & $71,2 \pm 5,3 \mathrm{~b}$ \\
Pluviosidade durante o período experimental $(\mathrm{mm})$ & $64,0 \mathrm{a}$ & $97,0 \mathrm{~b}$ \\
\hline
\end{tabular}

Médias seguidas por letras diferentes na mesma linha diferem entre si $(\mathrm{P}<0,01)$ pelo teste $\mathrm{F}$.

Vacas da raça Gir e Nelore apresentaram padrão de duas a quatro ondas de crescimento folicular, independentemente da estação do ano. As porcentagens de ciclos estrais com duas e três ondas foliculares foram 24,0 e $68,0 \%$ para a raça Gir e 26,7 e $66,7 \%$ para a raça Nelore, respectivamente $(\mathrm{P}>0,05)$. Somente duas vacas da raça Gir $(8 \%)$ e uma Nelore $(6,6 \%)$ apresentaram quatro ondas foliculares. Estes resultados demonstram que vacas de ambas as raças apresentam predominância de ciclos estrais com três ondas foliculares, similar aos estudos de Gambini et al. (1998) em vacas da raça Gir. Apesar de o experimento ter sido delineado para estudar o efeito da estação do ano sobre a dinâmica folicular, as análises mostraram não haver essa influência sobre as características avaliadas. Resultados da literatura indicam que existe variação na proporção de ciclos estrais com duas ou três

\footnotetext{
${ }^{1}$ Aloka SSD-500, Tokio, Japão
} 
Dinâmica folicular e momento da ovulação...

ondas foliculares entre animais de raças européias e zebuínas. Em todos os trabalhos com zebuínos, verifica-se a maior percentagem de ciclos estrais com duas ou três ondas foliculares, e menor freqüência com uma e quatro ondas (Rhodes et al., 1995a).

Apesar de alguns parâmetros como o diâmetro dos folículos dominantes e subordinados e área do corpo lúteo serem considerados menores em animais zebuínos do que naqueles de raças européias (Pathiraja et al., 1986; Figueiredo et al., 1995), a dinâmica folicular parece ser influenciada pelos mesmos fatores. Por isso, serão discutidas as peculiaridades do crescimento e da regressão dos folículos ovarianos sem que haja apenas comparações entre raças.

Algumas características das ondas de crescimento folicular de vacas das raças Gir e Nelore são apresentadas na Tab. 2.

Tabela 2. Características reprodutivas e de dinâmica folicular de vacas das raças Gir e Nelore

\begin{tabular}{|c|c|c|c|c|}
\hline \multirow{2}{*}{$\begin{array}{l}\text { Características reprodutiva } \\
\text { e de dinâmica folicular }\end{array}$} & \multicolumn{2}{|c|}{ Gir } & \multicolumn{2}{|c|}{ Nelore } \\
\hline & 2 ondas $\left(n=6^{*}\right)$ & 3 ondas $(\mathrm{n}=17 *)$ & 2 ondas $(n=4 *)$ & 3 ondas $\left(n=10^{*}\right)$ \\
\hline Intervalo estral (dias) & $18,6 \pm 0,8 \mathrm{a}$ & $20,6 \pm 1,3 \mathrm{~b}$ & $18,7 \pm 0,8 \mathrm{a}$ & $20,7 \pm 1,0 \mathrm{~b}$ \\
\hline \multicolumn{5}{|l|}{ Duração do crescimento (dias) } \\
\hline $1^{\circ}$ folículo & $5,8 \pm 1,2 \mathrm{aA}$ & $6,3 \pm 1,5 \mathrm{aA}$ & $8,0 \pm 1,4 \mathrm{aA}$ & $6,3 \pm 1,5 \mathrm{bA}$ \\
\hline $2^{\mathrm{Q}}$ folículo & $9,3 \pm 1,0 \mathrm{aB}$ & $4,6 \pm 1,6 \mathrm{bB}$ & $9,0 \pm 0,8 \mathrm{aA}$ & $5,4 \pm 1,4 \mathrm{bA}$ \\
\hline $3^{0}$ folículo & ------ & $6,1 \pm 1,4 \mathrm{~A}$ & ------ & $6,1 \pm 0,7 \mathrm{~A}$ \\
\hline \multicolumn{5}{|l|}{ Dia da detecção da onda** } \\
\hline $1^{\underline{a}}$ onda & $0,8 \pm 0,4 \mathrm{a}$ & $0,5 \pm 0,7 \mathrm{a}$ & $0,8 \pm 1,0 \mathrm{a}$ & $0,5 \pm 0,5 \mathrm{a}$ \\
\hline $2^{\underline{a}}$ onda & $9,2 \pm 0,8 \mathrm{a}$ & $8,4 \pm 1,4 \mathrm{a}$ & $9,8 \pm 1,0 \mathrm{a}$ & $8,1 \pm 0,9 b$ \\
\hline $3^{\underline{a}}$ onda & ------ & $14,3 \pm 1,8$ & ------ & $14,6 \pm 1,3$ \\
\hline \multicolumn{5}{|l|}{ Dia da divergência folicular } \\
\hline $1^{\underline{a}}$ onda & $3,7 \pm 0,8 \mathrm{aA}$ & $3,4 \pm 1,1 \mathrm{aA}$ & $5,5 \pm 0,6 \mathrm{aA}$ & $3,8 \pm 1,8 \mathrm{aA}$ \\
\hline $2^{\underline{a}}$ onda & $12,0 \pm 2,3 \mathrm{aB}$ & $11,1 \pm 2,0 \mathrm{aB}$ & $13,0 \pm 2,2 \mathrm{aB}$ & $13,3 \pm 3,7 \mathrm{aB}$ \\
\hline $3^{\mathrm{a}}$ onda & ------ & $17,0 \pm 2,0 \mathrm{C}$ & ------ & $16,3 \pm 1,6 \mathrm{C}$ \\
\hline
\end{tabular}

A duração média do intervalo de estros foi menor para ciclos com duas ondas, quando comparado com ciclos com três ondas de crescimento folicular $(\mathrm{P}<0,05)$, já que o número de ondas está relacionado com o comprimento do ciclo estral e com a duração da fase lútea (Fortune, 1993). Neste estudo, as vacas com quatro ondas foliculares apresentaram maiores médias para os intervalos de estro (25 dias) do que nas vacas com duas (18 dias) e três (20 dias) ondas foliculares.

Tanto nos ciclos de duas quanto nos de três ondas foliculares, o surgimento da primeira onda ocorreu entre os dias 0 (estro) e 1; a segunda, entre os dias 8,1 e 9,8; para os ciclos com três ondas, a terceira emergiu ao redor do dia 14. Nos ciclos de duas ondas, a segunda onda folicular ocorreu mais tardiamente na raça Nelore e não diferiu nas vacas da raça Gir $(\mathrm{P}>0,05)$ em relação aos ciclos com três ondas. Estudos com vacas da raça Gir têm demonstrado o surgimento de ondas nos dias 1,5 e
10,0 e 1,0, 9,4 e 14,6, respectivamente, nos ciclos com duas e três ondas foliculares (Gambini et al., 1998); nas vacas da raça Nelore, nos dias 1,5 e 12,0 e 1,5, 9,1 e 15,4 (Figueiredo et al., 1997).

Em média, dois a quatro dias após a detecção da onda folicular, independentemente do número de ondas, acontece a separação das curvas de crescimento entre o folículo dominante e seus subordinados (divergência ou seleção folicular). A seleção folicular nos bovinos é o momento no qual o folículo estrógeno-ativo promove seu crescimento e inibe o crescimento dos demais. Parece que o processo de divergência folicular é passivo, sendo o primeiro folículo a adquirir receptores para LH nas células da granulosa aquele que será selecionado para ser dominante (Wiltbank, 1998).

As características dos folículos dominantes de vacas com padrão de duas e três ondas são apresentadas na Tab. 3 . 
Borges et al.

Tabela 3. Características dos folículos dominantes de vacas das raças Gir e Nelore com padrão de duas ou três ondas foliculares

\begin{tabular}{|c|c|c|c|c|}
\hline \multirow{2}{*}{ Característica } & \multicolumn{2}{|c|}{ Gir } & \multicolumn{2}{|c|}{ Nelore } \\
\hline & 2 ondas $\left(n=6^{*}\right)$ & 3 ondas $\left(\mathrm{n}=17^{*}\right)$ & 2 ondas $(\mathrm{n}=4 *)$ & 3 ondas $\left(\mathrm{n}=10^{*}\right)$ \\
\hline \multicolumn{5}{|c|}{ Taxa de crescimento $(\mathrm{mm} / \mathrm{dia})$} \\
\hline $1^{\circ}$ folículo & $1,4 \pm 0,2 \mathrm{aA}$ & $1,4 \pm 0,3 \mathrm{aA}$ & $1,2 \pm 0,1 \mathrm{aA}$ & $1,4 \pm 0,3 \mathrm{aA}$ \\
\hline $2^{\circ}$ folículo & $1,2 \pm 0,2 \mathrm{aA}$ & $1,3 \pm 0,4 \mathrm{aA}$ & $1,1 \pm 0,1 \mathrm{aA}$ & $1,2 \pm 0,2 \mathrm{aA}$ \\
\hline $3^{\circ}$ folículo & ------ & $1,4 \pm 0,3 \mathrm{~A}$ & ------ & $1,4 \pm 0,3 \mathrm{aA}$ \\
\hline \multicolumn{5}{|c|}{ Diâmetro máximo (mm) } \\
\hline $1^{\mathrm{o}}$ folículo & $13,2 \pm 1,3 \mathrm{aA}$ & $12,9 \pm 2,2 \mathrm{aA}$ & $13,5 \pm 1,3 \mathrm{aA}$ & $13,0 \pm 1,8 \mathrm{aA}$ \\
\hline $2^{\underline{0}}$ folículo & $15,0 \pm 1,4 \mathrm{aA}$ & $9,8 \pm 1,5 \mathrm{bB}$ & $14,5 \pm 1,0 \mathrm{aA}$ & $10,6 \pm 0,8 \mathrm{bB}$ \\
\hline $3^{\circ}$ folículo & ----- & $12,8 \pm 1,4 \mathrm{~A}$ & ----- & $13,2 \pm 2,1 \mathrm{~A}$ \\
\hline \multicolumn{5}{|c|}{ Dia do diâmetro máximo } \\
\hline $1^{\circ}$ folículo & $6,7 \pm 1,0 \mathrm{aA}$ & $6,9 \pm 1,5 \mathrm{aA}$ & $8,8 \pm 2,2 \mathrm{aA}$ & $6,8 \pm 1,1 \mathrm{bA}$ \\
\hline $2^{\circ}$ folículo & $18,5 \pm 0,8 \mathrm{aB}$ & $12,9 \pm 1,9 \mathrm{bB}$ & $19,0 \pm 1,4 \mathrm{aB}$ & $13,5 \pm 1,6 \mathrm{bB}$ \\
\hline $3^{\circ}$ folículo & ----- & $20,5 \pm 1,7 \mathrm{C}$ & ----- & $20,7 \pm 1,3 \mathrm{C}$ \\
\hline \multicolumn{5}{|c|}{ Duração da dominância (dias) } \\
\hline $1^{\circ}$ folículo & $5,5 \pm 1,2 \mathrm{aA}$ & $5,0 \pm 1,4 \mathrm{aA}$ & $5,0 \pm 1,4 \mathrm{aA}$ & $4,6 \pm 1,2 \mathrm{aA}$ \\
\hline $2^{\circ}$ folículo & $6,5 \pm 2,3 \mathrm{aA}$ & $3,3 \pm 1,3 \mathrm{bB}$ & $4,5 \pm 1,4 \mathrm{aB}$ & $4,1 \pm 1,6 \mathrm{aA}$ \\
\hline $3^{\circ}$ folículo & ------ & $3,5 \pm 1,5 \mathrm{~B}$ & ------ & $4,1 \pm 1,2 \mathrm{~A}$ \\
\hline \multicolumn{5}{|c|}{ Início da atresia (dia) } \\
\hline $1^{\circ}$ folículo & $10,0 \pm 2,5 \mathrm{a}$ & $7,6 \pm 1,7 \mathrm{bA}$ & $11,5 \pm 1,7 \mathrm{a}$ & $8,1 \pm 1,3 \mathrm{bA}$ \\
\hline $2^{\underline{0}}$ folículo & ------ & $13,8 \pm 2,7 \mathrm{~B}$ & ------ & $14,8 \pm 2,4 \mathrm{~B}$ \\
\hline $3^{0}$ folículo & ------ & ------ & ------ & - \\
\hline \multicolumn{5}{|c|}{ Taxa de atresia $(\mathrm{mm} / \mathrm{dia})$} \\
\hline $1^{\circ}$ folículo & $1,0 \pm 0,3 \mathrm{a}$ & $1,1 \pm 0,3 \mathrm{aA}$ & $1,0 \pm 0,2 \mathrm{a}$ & $0,8 \pm 0,2 \mathrm{aA}$ \\
\hline $2^{\circ}$ folículo & ----- & $1,0 \pm 0,3 \mathrm{~A}$ & ------ & $0,8 \pm 0,3 \mathrm{~A}$ \\
\hline $3^{\circ}$ folículo & ------ & ------ & ------ & - \\
\hline \multicolumn{5}{|c|}{ Duração da atresia (dias) } \\
\hline $1^{\underline{0}}$ folículo & $9,5 \pm 2,1 \mathrm{a}$ & $8,5 \pm 3,0 \mathrm{aA}$ & $9,5 \pm 1,9 \mathrm{a}$ & $10,6 \pm 2,2 \mathrm{aA}$ \\
\hline $2^{\underline{0}}$ folículo & ------ & $5,4 \pm 1,7 \mathrm{~B}$ & ------ & $7,3 \pm 1,4 \mathrm{~B}$ \\
\hline $3^{\circ}$ folículo & ------ & ------ & ------ & ------ \\
\hline
\end{tabular}

Após o surgimento de qualquer uma das ondas foliculares, o folículo dominante cresceu por cerca de 4,6 a 9,3 dias (Tab. 2) a uma taxa de crescimento de 1,1 a $1,4 \mathrm{~mm} /$ dia até atingir o diâmetro máximo entre 9,8 e 15,0mm (Tab. 3). É importante ressaltar que nos ciclos estrais com padrão de três ondas foliculares, o segundo folículo dominante é menor que o da primeira e terceira ondas, apesar de que nos da raça Nelore não houve efeito na duração do crescimento. Uma possível explicação para a diferença nos diâmetros foliculares é a concentração plasmática de progesterona, na qual a primeira onda folicular coincide com a baixa concentração (corpo lúteo em formação), insuficiente para efetuar um feedback negativo hipotalâmicohipofisário satisfatório que impeça a liberação de hormônio luteinizante. Pulsos de baixa freqüência de LH (entre 2 e $3 \mathrm{ng} / \mathrm{ml}$ ), observados durante a fase lútea do ciclo estral, levam à regressão do folículo dominante e ao início de novas ondas foliculares (Sávio et al., 1992). A segunda onda inicia-se na presença de um corpo lúteo totalmente formado e que secreta elevada concentração de progesterona, suficiente para inibir a liberação de LH. O menor diâmetro do folículo da segunda onda também pode ser devido à menor duração de crescimento desses (4,6 e 5,4 dias) quando comparado com os folículos da primeira (6,3 dias) e terceira ondas (6,1 dias), o que não ocorreu nos animais da raça Nelore. A terceira onda coincide com a redução da secreção de progesterona pelo corpo lúteo, resultando no aumento da freqüência de pulsos 
de LH até um pico de 30 a $35 \mathrm{ng} / \mathrm{ml}$. Nessa fase é que se verificam o rápido crescimento folicular e a ovulação (Sávio et al., 1992).

Após a divergência, o folículo dominante é selecionado para continuar crescendo e, neste experimento, apresentou período de dominância de 3,3 a 6,5 dias. Após a perda de sua funcionalidade, o folículo dominante iniciou $\mathrm{o}$ processo de atresia ou regressão à taxa de 0,8 a $1,1 \mathrm{~mm} /$ dia. $\mathrm{O}$ tempo necessário para atingir o diâmetro final de 4 a $5 \mathrm{~mm}$ variou de 5,4 a 10,6 dias. O diâmetro dos folículos ovulatórios (de
12,8 a $15 \mathrm{~mm}$ ) foi aproximadamente o mesmo do encontrado na raça Guzerá $(11,4$ a $16,2 \mathrm{~mm}$; Fonseca, 2000) e menor do que os de animais de raças européias (15 a 20mm; Savio et al., 1988). O menor valor com relação aos taurinos pode estar relacionado à menor taxa de crescimento dos folículos dominantes de vacas zebuínas $(0,8 \mathrm{~mm} /$ dia de Figueiredo et al., 1997 versus 2,0mm/dia de Murphy et al., 1990).

As características dos folículos subordinados são apresentadas na Tab. 4.

Tabela 4. Características dos folículos subordinados de vacas das raças Gir e Nelore com padrão de duas ou três ondas foliculares

\begin{tabular}{|c|c|c|c|c|}
\hline \multirow{2}{*}{ Característica } & \multicolumn{2}{|c|}{ Gir } & \multicolumn{2}{|c|}{ Nelore } \\
\hline & 2 ondas $\left(\mathrm{n}=6^{*}\right)$ & 3 ondas $(n=17 *)$ & 2 ondas $(n=4 *)$ & 3 ondas $\left(\mathrm{n}=10^{*}\right)$ \\
\hline \multicolumn{5}{|c|}{ Diâmetro máximo (mm) } \\
\hline $1^{\circ}$ folículo & $7,7 \pm 0,8 \mathrm{aA}$ & $7,1 \pm 1,2 \mathrm{aA}$ & $8,0 \pm 0,8 \mathrm{aA}$ & $7,1 \pm 1,4 \mathrm{aA}$ \\
\hline $2^{\underline{0}}$ folículo & $7,3 \pm 2,0 \mathrm{aA}$ & $6,5 \pm 1,2 \mathrm{aA}$ & $7,0 \pm 0,8 \mathrm{aA}$ & $6,4 \pm 1,6 \mathrm{aA}$ \\
\hline $3^{\mathrm{o}}$ folículo & ------ & $7,4 \pm 1,2 \mathrm{~A}$ & ------ & $6,6 \pm 1,5 \mathrm{~A}$ \\
\hline \multicolumn{5}{|c|}{ Dia do diâmetro máximo } \\
\hline $1^{\mathrm{O}}$ folículo & $3,7 \pm 0,8 \mathrm{aA}$ & $3,2 \pm 1,0 \mathrm{aA}$ & $5,5 \pm 0,6 \mathrm{aA}$ & $4,2 \pm 2,0 \mathrm{aA}$ \\
\hline $2^{\underline{0}}$ folículo & $11,8 \pm 2,2 \mathrm{aB}$ & $11,1 \pm 2,0 \mathrm{aB}$ & $13,0 \pm 2,2 \mathrm{aB}$ & $10,1 \pm 1,4 \mathrm{bB}$ \\
\hline $3^{\mathrm{o}}$ folículo & ----- & $17,0 \pm 1,9 \mathrm{C}$ & ----- & $16,8 \pm 2,7 \mathrm{C}$ \\
\hline \multicolumn{5}{|c|}{ Persistência folicular (dias) } \\
\hline $1^{\circ}$ folículo & $7,2 \pm 0,8 \mathrm{aA}$ & $6,4 \pm 1,8 \mathrm{aA}$ & $9,5 \pm 1,9 \mathrm{aA}$ & $7,5 \pm 2,6 \mathrm{aA}$ \\
\hline $2^{\mathrm{o}}$ folículo & $6,0 \pm 3,2 \mathrm{aA}$ & $5,6 \pm 2,2 \mathrm{aA}$ & $7,8 \pm 1,0 \mathrm{aA}$ & $5,6 \pm 2,6 \mathrm{aA}$ \\
\hline $3^{\mathrm{o}}$ folículo & ------ & $5,9 \pm 1,5 \mathrm{~A}$ & ------ & $4,7 \pm 1,9 \mathrm{~A}$ \\
\hline \multicolumn{5}{|c|}{ Duração do crescimento (dias) } \\
\hline $1^{\circ}$ folículo & $2,8 \pm 1,3 \mathrm{aA}$ & $2,7 \pm 1,1 \mathrm{aA}$ & $4,7 \pm 1,5 \mathrm{aA}$ & $3,7 \pm 2,0 \mathrm{aA}$ \\
\hline $2^{\circ}$ folículo & $2,7 \pm 2,3 \mathrm{aA}$ & $2,6 \pm 1,4 \mathrm{aA}$ & $3,3 \pm 1,7 \mathrm{aA}$ & $1,8 \pm 0,8 \mathrm{aB}$ \\
\hline $3^{\mathrm{o}}$ folículo & ------ & $2,8 \pm 1,0 \mathrm{~A}$ & ------ & $2,2 \pm 1,4 \mathrm{~A}$ \\
\hline \multicolumn{5}{|c|}{ Taxa de crescimento (mm/dia) } \\
\hline $1^{\circ}$ folículo & $1,2 \pm 0,4 \mathrm{aA}$ & $1,1 \pm 0,3 \mathrm{aA}$ & $0,9 \pm 0,4 \mathrm{aA}$ & $1,0 \pm 0,4 \mathrm{aA}$ \\
\hline $2^{\underline{0}}$ folículo & $1,6 \pm 0,5 \mathrm{aA}$ & $0,9 \pm 0,3 \mathrm{aA}$ & $1,2 \pm 0,6 \mathrm{aA}$ & $1,5 \pm 0,9 \mathrm{aB}$ \\
\hline $3^{\mathrm{o}}$ folículo & ----- & $1,2 \pm 0,4 \mathrm{~A}$ & ----- & $1,2 \pm 0,5 \mathrm{~A}$ \\
\hline \multicolumn{5}{|c|}{ Início da atresia (dia) } \\
\hline $1^{\circ}$ folículo & $3,8 \pm 0,8 \mathrm{aA}$ & $4,0 \pm 1,3 \mathrm{aA}$ & $5,8 \pm 1,0 \mathrm{aA}$ & $5,6 \pm 1,8 \mathrm{aA}$ \\
\hline $2^{\circ}$ folículo & $12,7 \pm 2,2 \mathrm{aB}$ & $12,0 \pm 2,0 \mathrm{aB}$ & $14,5 \pm 1,3 \mathrm{aB}$ & $11,5 \pm 1,3 \mathrm{bB}$ \\
\hline $3^{\circ}$ folículo & ------ & $17,8 \pm 2,1 \mathrm{C}$ & ------ & $16,9 \pm 1,5 \mathrm{C}$ \\
\hline \multicolumn{5}{|c|}{ Taxa de atresia $(\mathrm{mm} / \mathrm{dia})$} \\
\hline $1^{\underline{0}}$ folículo & $1,0 \pm 0,5 \mathrm{aA}$ & $1,1 \pm 0,4 \mathrm{aA}$ & $1,1 \pm 0,5 \mathrm{aA}$ & $1,4 \pm 0,4 \mathrm{aA}$ \\
\hline $2^{\underline{0}}$ folículo & $1,1 \pm 0,5 \mathrm{aA}$ & $1,1 \pm 0,3 \mathrm{aA}$ & $1,1 \pm 0,3 \mathrm{aA}$ & $1,0 \pm 0,4 \mathrm{aA}$ \\
\hline $3^{-}$folículo & ------ & $1,3 \pm 0,4 \mathrm{~A}$ & ------ & $1,2 \pm 0,5 \mathrm{~A}$ \\
\hline \multicolumn{5}{|c|}{ Duração da atresia (dias) } \\
\hline $1^{\circ}$ folículo & $4,2 \pm 1,2 \mathrm{aA}$ & $2,9 \pm 1,3 \mathrm{bA}$ & $4,3 \pm 2,9 \mathrm{aA}$ & $2,4 \pm 1,2 \mathrm{aA}$ \\
\hline $2^{\underline{0}}$ folículo & $2,5 \pm 1,0 \mathrm{aB}$ & $2,0 \pm 1,1 \mathrm{aA}$ & $3,0 \pm 1,4 \mathrm{aA}$ & $2,2 \pm 1,5 \mathrm{aA}$ \\
\hline $3^{\circ}$ folículo & ----- & $2,4 \pm 1,1 \mathrm{~A}$ & ----- & $1,9 \pm 1,1 \mathrm{~A}$ \\
\hline
\end{tabular}


O diâmetro máximo dos folículos subordinados foi de 6,4 a $8,0 \mathrm{~mm}$, alcançado após um período médio de crescimento de 2,6 dias, e sua persistência foi de 4,7 a 9,5 dias. Estes valores estão próximos dos encontrados em animais da raça Nelore (Figueiredo et al., 1997) e Holandesa (Fortune, 1994). A regressão dos subordinados iniciou-se cerca de três a cinco dias após o surgimento da onda folicular. O menor diâmetro

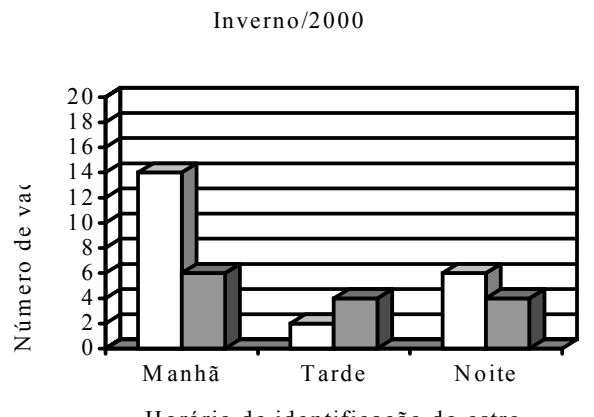

Horário de identificação do estro

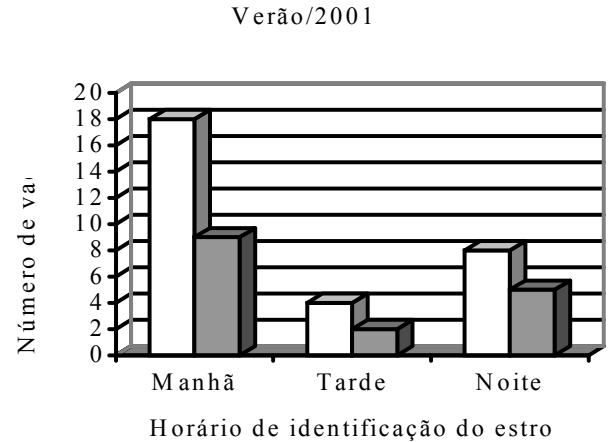

dos subordinados é atribuído à inibição exercida pelo folículo dominante, por meio da redução da concentração de FSH, insuficiente para manter o desenvolvimento dos folículos subordinados (Driancourt et al., 1991).

A distribuição dos estros e ovulações está na Fig. 1.
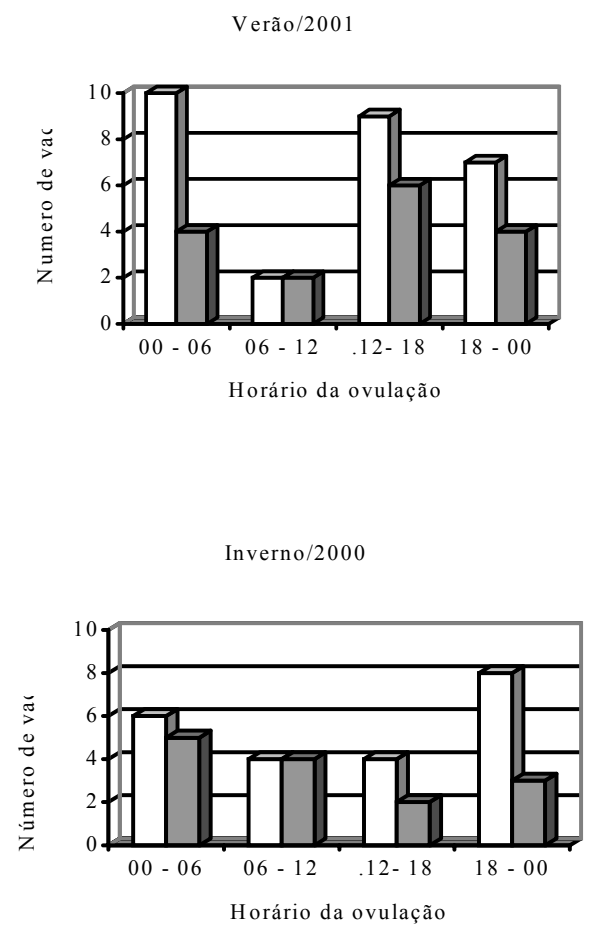

Figura 1. Horário de identificação do estro e de ovulação a partir do momento da detecção do estro em vacas das raças Gir (branco) e Nelore (cinza), em duas estações do ano: inverno (Gir= 22 e Nelore $=14)$ e verão $(\mathrm{Gir}=30$ e Nelore $=16)$.

Verificou-se maior ocorrência de estros no turno da manhã e início da noite nas duas estações, e menor ocorrência entre as 12 e 13 horas. Isto mostra a associação do início do estro com as horas de temperaturas mais amenas do dia. Mesmo nos meses de clima mais frio, a distribuição dos estros manteve-se pela manhã e à tarde, contradizendo a explicação de LamotheZavaleta et al. (1991) de que no inverno (temperatura média de $22,3^{\circ} \mathrm{C}$ ) as baixas temperaturas poderiam impedir a expressão sexual. O manejo de animais zebuínos é diferenciado por características próprias da subespécie, tais como comportamento (Pinheiro et al., 1998), intensidade, duração e horário de manifestação do estro (Galina et al., 1990; Lamothe-Zavaleta et al., 1991). Neste experimento, todos os animais foram identificados em estro característico, contradizendo as dificuldades expostas por aqueles autores. As manifestações ocorreram mais nas primeiras horas da manhã $(57,3 \%)$ e 
entre o final da tarde e início da noite $(28,0 \%)$ e menos à tarde $(14,6 \%)$. Isto demonstra que a eficiência na detecção do estro depende do treinamento do observador, bem como da manutenção da rotina dos horários de observação. A não observação noturna pode ser a causa do aumento de ocorrências pela manhã.
O momento de ovulação ocorreu, em média, cerca de 23,1 $\pm 5,6$ horas após a detecção do estro e não diferiu entre as raças e estações do ano (Tab. 5). Um animal da raça Nelore apresentou estro prolongado além da média normalmente verificada e foi excluído das análises estatísticas.

Tabela 5. Médias e desvios-padrão para o intervalo (horas) do início do estro à ovulação de vacas das raças Gir e Nelore, dentro de duas estações do ano

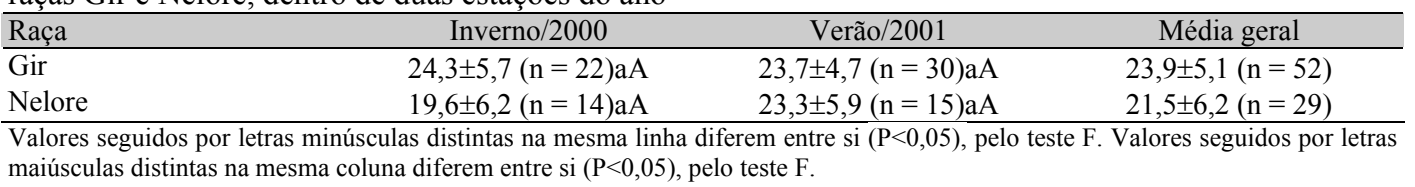

Estes intervalos são inferiores aos encontrados nas raças Gir (28h; Viana et al., 1999), Nelore (26,8h; Pinheiro et al., 1998) e vacas mestiças (32h; Wettemann et al., 1998). Isto pode ter ocorrido porque nesses trabalhos utilizou-se observação ininterrupta do estro durante as $24 \mathrm{~h}$ do dia, o que não aconteceu neste experimento. Nele adotou-se a prática de observação de estro similar à normalmente utilizada nas propriedades, diferindo apenas no rigor dos horários de detecção matinal e noturna. Mesmo assim, esse manejo mostrou-se bastante eficiente.

\section{CONCLUSÕES}

A dinâmica folicular de vacas das raças Gir e Nelore é similar à de animais europeus. Não foi encontrada dificuldade na identificação do estro dos animais, o que contraria os resultados de autores que justificam o baixo desempenho reprodutivo de animais zebuínos como conseqüência de problemas de comportamento, intensidade, duração e horário de manifestação do estro. A utilização de um bom manejo de observação de estro é necessária para se maximizar as respostas dos animais nos programas de inseminação artificial. A manifestação do estro ocorreu nas primeiras horas da manhã e ao anoitecer, sendo importante a monitoração da atividade estral durante maior período de tempo. O momento de ovulação quanto ao horário de deteç̧ão do estro ocorre mais precocemente que em animais de raças européias.

\section{REFERÊNCIAS BIBLIOGRÁFICAS}

ADAMS, G.P.; MATTERI, R.L.; KASTELIC, J.P. et al. Association between surges of FSH and emergence of follicular waves in heifers. J. Reprod. Fertil., v.94, p.177-188, 1992.

DRIANCOURT, M.A. Follicular dynamics in sheep and cattle. Theriogenology, v.35, p.55-71, 1991.

FERREIRA, A.M. Efeito da amamentação e do nivel nutricional na atividade ovariana de vacas mestiças leiteiras. 1990. 134f. Tese (Doutorado em Zootecnia) - Universidade Federal de Viçosa, Viçosa, MG.

FIGUEIREDO, R.A.; BARROS, C.M.; PINHEIRO, O.L. et al. Ovarian follicular dynamics in Nelore breed (Bos indicus) cattle. Theriogenology, v.47, p.1489-1505, 1997.

FIGUEIREDO, R.A.; BARROS, C.M.; ROCHA, G.P. et al. Prevalência de duas ondas de crescimento folicular ovariano em vacas da raça Nelore. Rev. Bras. Reprod. Anim., v.19, p.200-211, 1995.

FONSECA, L.M. Aspiração folicular em novilhas da raça Guzerá submetidas a tratamento hormonal com rBST associada ao FSH e dinâmica folicular durante o ciclo estral. 2000. 109f. Dissertação (Mestrado em Produção Animal) - Universidade Estadual do Norte Fluminense, Campos dos Goytacazes, RJ.

FORTUNE, J.E. Follicular dynamics during the bovine estrus cycle: a limiting factor in improvement of fertility? Anim. Reprod. Sci., v.33, p.111-125, 1993. 
FORTUNE, J.E. Ovarian follicular growth and development in mammals. Biol. Reprod., v.50, p.225-232, 1994.

GALINA, C.S.; ARTHUR, G.H. Review on cattle reproduction in the tropics. Part 4: Oestrus cycles. Anim. Breed. Abstr., v.58, p.697-707, 1990.

GAMBINI, A.L.G.; MOREIRA, M.B.P.; CASTILHO, C. et al. Desenvolvimento folicular e sincronização da ovulação em vacas da raça Gir. Rev. Bras. Reprod. Anim., v.22, p.201-210, 1998.

GINTHER, O.J. Selection of the dominant follicle in cattle and horses. Anim. Reprod. Sci., v.60-61, p.61-79, 2000.

GINTHER, O.J.; KNOPF, L.; KASTELIC, J.P. Temporal associations among ovarian events in cattle during oestrus cycles with two and three follicular waves. J. Reprod. Fertil., v.87, p.223230, 1989.

LAMOTHE-ZAVALETA, C.; FREDERICKSSON, G.; KINDAHL, H. Reproductive performance of zebu cattle in Mexico. 1. Sexual behavior and seasonal influence on estrous cyclicity. Theriogenology, v.36, p.887-896, 1991.

MURPHY, M.G.; BOLAND, M.P.; ROCHE, J.F. Pattern of follicular growth and resumption of ovarian activity in post-partum beef suckler cows. J. Reprod. Fertil., v.90, p.523-533, 1990.

PATHIRAJA, N.; OYEDIPE, E.O.; VOH Jr., A.A. et al.. Accuracy of retal palpation in the diagnosis of corpora lutea in Zebu cows. Br. Vet. J., v.142, p.467-471, 1986.

PINHEIRO, O.L.; BARROS, C.M.; FIGUEIREDO, R.A. et al. Estrous behaviour and the estrus-toovulation interval in Nelore cattle (Bos indicus) with natural estrus or estrus induced with prostaglandin $F_{2 \alpha}$ or Norgestomet and estradiol valerate. Theriogenology, v.49, p.667-681, 1998.

PLASSE, D.; WARNICK, A.C., KOGER, M. Reproductive behavior of Bos indicus females in a subtropical environment. IV. Length of estrous cycle, duration of estrus, time of ovulation, fertilization and embryo survival in Grade Brahman heifers. J. Anim. Sci., v.30, p.63-71, 1970.

RANDEL, R.D. Unique reproductive traits of Brahman and Brahman based cows. In: FIELDS, M.J; SAND, R.S. Factors affecting calf crop. Boca Raton, 1994. p.23-43.

RHODES, F.M.; DE'ATH, G.; ENTWISTLE, K.W. Animal and temporal effects on follicular dynamics in Brahman heifers. Anim. Reprod. Sci., v.38, p.265-277, 1995 b.

RHODES, F.M.; FITZPATRICK, L.A.; ENTWISTLE, K.W. et al. Sequential changes in ovarian follicular dynamics in Bos indicus heifers before and after nutritional anoestrus. J. Reprod. Fertil., v.104, p.41-49, 1995a.

RHODES, R.C.; RANDEL, R.D.; LONG, C.R. Corpus luteum function in the bovine: in vivo and in vitro evidence for both a seasonal and breedtype effect. J. Anim. Sci., v.55, p.159-167, 1982.

SAVIO, J.D.; KEENAN, L.; BOLAND, M.P. et al. Pattern of growth of dominant follicles during the estrus cycle of heifers. J. Reprod. Fertil., v.83, p.663-671, 1988.

SAVIO, J.D.; THATCHER, W.W.; MORRIS,G.R. et al. Terminal follicular development and fertility in cattle is regulated by concentration of plasma progesterone. INTERNATIONAL CONGRESS ON ANIMAL REPRODUCTION, 12., 1992, The Hague. Proceedings..., The Hague : ICAR, 1992. v.2, p.999-1002.

SISTEMA de análises estatísticas e genéticas SAEG. Viçosa: UFV, 1997. 59p.

STAHRINGER, R.C.; NEUENDORFF, D.A.; RANDEL, R.D. Seasonal variations in characteristics of estrous cycles in pubertal Brahman heifers. Theriogenology, v.34, p.407-416, 1990.

VALLE, E.R.; ENCARNAÇÃO, R.O.; SCHENK, J.A.P. et al. Duração do cio e momento de ovulação em vacas Nelore. Rev. Soc. Bras. Zootec., v.23, p.852-858, 1994.

VIANA, J.H.M.; PIRES, M.F.A.; CAMARGO, L.S.A. et al. Dinâmica folicular no período préovulatório, estro e momento de ovulação em vacas Gir. Rev. Bras. Reprod. Anim., v.23, p.159-160, 1999.

WETTEMANN, R.P.; LOOPER, M.L.; WARNER, M.L. et al. Estrous behavior and time of ovulation in beef cows during the summer. Anim. Sci. Res. Reprod., p.227-231, 1998.

WILTBANK, M.C. Regulation of the ovary in cattle. Curso Novos enfoques na produção $e$ reprodução de bovinos, 2., Passos, MG. Anais... Passos: [s.n.], 1998. p.1-13.

ZEITOUN, M.M.; RODRIGUEZ, H.F.; RANDEL, R.D. Effect of season on ovarian follicular dynamics in Brahman cows. Theriogenology, v.45, p.1577-1581, 1996. 